\title{
Correction to: Arthrobacter wenxiniae sp. nov., a novel plant growth-promoting rhizobacteria species harbouring a carotenoids biosynthetic gene cluster
}

\author{
Yu-chen Sun • Pengbo Sun • Jing Xue • \\ Yunpeng Du • Hui Yan • Li-wei Wang • Xin-xin Yi • \\ Jian-guang Sun $\cdot$ Xiuhai Zhang $\cdot$ Jun-lian Gao
}

Published online: 7 March 2022

(C) Springer Nature Switzerland AG 2022

\section{Correction to:}

Antonie van Leeuwenhoek(2022)

https://doi.org/10.1007/s10482-021-01701-9

In the original publication of the article, there is a mistake in the first citation. It is written as follows: Antonio J, Fernández G, Manuel FL (2018) Wholegenome sequences of two Arthrobacter strains isolated from a Holm Oak rhizosphere affected by wildfire. Genom Announc 6(11): e00071-18-e00071-18. The givenname and surnames of the authors is not written well. It should be: Fernández-González AJ, Lasa AV, Fernández-López M (2018) Whole-Genome

Sequences of Two Arthrobacter Strains Isolated from a Holm Oak Rhizosphere Affected by Wildfire. Genom Announc 6(11):e00071-18-e00071-18.

Further, the first citation quoted in the first paragragh of the introduction has also been written incorrectly. While the citation published reads "Antonio et al. 2018", the correct citation should now read as “Fernández-López et al. 2018”.

Publisher's Note Springer Nature remains neutral with regard to jurisdictional claims in published maps and institutional affiliations.

The original article can be found online at https://doi.org/ 10.1007/s10482-021-01701-9.

Y. Sun $\cdot$ J. Xue $\cdot$ Y. Du $\cdot$ L. Wang $\cdot$ X. Zhang $(\bowtie) \cdot$ J. Gao $(\bowtie)$

Institute of Grassland, Flowers and Ecology, Beijing Academy of Agriculture and Forestry Sciences,

Beijing 100097, People's Republic of China

e-mail: zhangxiuhai@baafs.net.cn

J. Gao

e-mail: gaojunlian@baafs.net.cn

Y. Sun $\cdot$ X. Yi

College of Food Science and Engineering, Beijing

University of Agriculture, Beijing 102206,

People's Republic of China

\author{
P. Sun \\ German Cancer Consortium(DKTK), German Cancer \\ Research Center(DKFZ), 69120 Heidelberg, Germany \\ H. Yan \\ College of Animal Science and Technology, \\ Hebei Agricultural University, Baoding 071001, \\ People's Republic of China \\ J. Sun \\ Key Laboratory of Microbial Resources, Ministry \\ of Agriculture/Institute of Agricultural Resources \\ and Regional Planning, Chinese Academy of Agricultural \\ Sciences, Beijing 100081, People's Republic of China
}

P. Sun

Division of Pediatric Neurooncology, German Cancer

Research Center (DKFZ), 69120 Heidelberg, Germany 